\title{
Judicialização do cuidado na infância e na adolescência: relato de experiência
}

\author{
Judicialization of care in childhood and adolescence: experience report \\ Judicialización del cuidado en la niñez y adolescencia: informe de experiencia
}

Recebido: 23/11/2021 | Revisado: 28/11/2021 | Aceito: 29/11/2021 | Publicado: 10/12/2021

\author{
Priscila dos Santos Góes \\ ORCID: https://orcid.org/0000-0002-7379-8768 \\ Universidade do Vale do Rio dos Sinos, Brasil \\ E-mail: priscila.goes.nutri@gmail.com \\ Vanessa Ruffatto Gregoviski \\ ORCID: https://orcid.org/0000-0003-2404-8714 \\ Universidade do Vale do Rio dos Sinos, Brasil \\ E-mail: vane.ruffatto2@hotmail.com \\ Marla Fernanda Kuhn \\ ORCID: https://orcid.org/0000-0001-9228-6226 \\ Vigilância em Saúde Porto Alegre, Brasil \\ E-mail: marlakuhn2016@gmail.com
}

\begin{abstract}
Resumo
Este manuscrito objetiva relatar a vivência de uma residente em saúde mental, que atuou em espaços de assistência à saúde e gestão em saúde, discutindo situações de judicialização do cuidado de crianças e adolescentes. Entende-se que a internação compulsória deles se constitui como um assunto de suma importância pelo aumento de casos e para elucidação dos fluxos de cuidado. É um relato qualitativo, transversal e exploratório, cujos instrumentos foram diários de campo e observação participante. A escrita versará sobre a experiência da residente em si, sem descrever serviços ou profissionais daqueles espaços. As percepções sinalizam a importância da educação permanente sobre o assunto, e consequente qualificação constante de profissionais que atuem tanto na assistência quanto na gestão em saúde. É perceptível o alto número de internação do público infanto-juvenil, especialmente àqueles que já vivenciaram grave violações de direitos humanos e/ou fazem uso de substâncias psicoativas, mesmo em situações não abusivas. A baixa articulação entre os atores de cuidado, bem como a dificuldade em aderência ao cuidado proposto a eles, ainda é um agravante não superado, refletindo na judicialização de infantes e jovens. Logo, a importância da residência consiste na sua estratégia de formação a profissionais que buscarão atuar na saúde pública e àqueles que já o fazem. O papel do residente se evidencia na potencialização de discussões teóricas que se aliem àquelas técnicas, promovendo reflexões críticas com análises participativas, percebendo incoerências e contradições na execução das políticas públicas de cuidado.
\end{abstract}

Palavras-chave: Saúde mental; Políticas públicas; Internação compulsória; Crianças; Adolescentes.

\begin{abstract}
This manuscript aims to report the experience of a mental health resident, who worked in health care and health management spaces, discussing situations of judicialization of child and adolescent care. It is understood that their compulsory hospitalization is a matter of paramount importance due to the increase in cases and to elucidate care flows. It is a qualitative, transversal and exploratory report, whose instruments were field diaries and participant observation. The writing will deal with the experience of the resident itself, without describing services or professionals in those spaces. The perceptions signal the importance of continuing education on the subject, and consequent constant qualification of professionals who work both in care and in health management. The high number of hospitalizations of children and adolescents is noticeable, especially those who have already experienced serious violations of human rights and/or use psychoactive substances, even in non-abusive situations. The low articulation between the care actors, as well as the difficulty in adhering to the care proposed to them, is still an unresolved aggravating factor, reflecting on the judicialization of infants and young people. Therefore, the importance of residency lies in its training strategy for professionals who will seek to work in public health and for those who already do so. The resident's role is evidenced in the potentialization of theoretical discussions that are combined with those techniques, promoting critical reflections with participatory analysis, realizing inconsistencies and contradictions in the implementation of public care policies.
\end{abstract}

Keywords: Mental health; Public policy; Compulsory hospitalization; Children; Adolescents.

\section{Resumen}

Este manuscrito tiene como objetivo relatar la experiencia de un residente de salud mental, que trabajó en los espacios de atención y gestión de la salud, discutiendo situaciones de judicialización de la atención a la niñez y adolescencia. 
Se entiende que su internación obligatoria es un asunto de suma importancia por el aumento de casos y para dilucidar los flujos asistenciales. Es un informe cualitativo, transversal y exploratorio, cuyos instrumentos fueron diarios de campo y observación participante. La redacción tratará de la experiencia del propio residente, sin describir los servicios ni los profesionales en esos espacios. Las percepciones señalan la importancia de la educación continua en el tema, y la consecuente cualificación constante de los profesionales que laboran tanto en el cuidado como en la gestión de la salud. Se nota el elevado número de hospitalizaciones de niños, niñas y adolescentes, especialmente de aquellos que ya han sufrido graves violaciones a los derechos humanos y / o consumen sustancias psicoactivas, incluso en situaciones no abusivas. La baja articulación entre los actores del cuidado, así como la dificultad para adherirse al cuidado que se les propone, sigue siendo un agravante no resuelto, reflejándose en la judicialización de la infancia y la juventud. Por tanto, la importancia de la residencia radica en su estrategia de formación para los profesionales que buscarán trabajar en salud pública y para los que ya lo hacen. El rol del residente se evidencia en la potencialización de discusiones teóricas que se combinan con esas técnicas, promoviendo reflexiones críticas con análisis participativo, reconociendo inconsistencias y contradicciones en la implementación de las políticas públicas de atención.

Palabras clave: Salud mental; Políticas públicas; Hospitalización obligatoria; Niños; Adolescentes.

\section{Introdução}

A partir da Lei nº 11.129 de 2005, promulgaram-se os programas de Residência Profissional na área da Saúde, especialização que proporciona aproximação e indissociabilidade entre serviços públicos de saúde e ensino dos acadêmicos/profissionais que dela fazem parte. A experiência relatada neste manuscrito se refere à vivência ocorrida em um programa de residência em saúde mental, pautado na Reforma Psiquiátrica como norteadora, baseando-se nos preceitos e princípios do Sistema Único de Saúde (SUS) e enfatizando a importância de um modelo assistencial voltado ao território, direitos humanos, acolhimento, vinculação e co-responsabilização (Brasil, 2001; Brasil, 2005; Mattos \& Gregoviski, 2020).

Entende-se que a Residência Multiprofissional em Saúde (RMS) traz aos profissionais que dela fazem parte, a possibilidade de formação em serviço, articulando saberes teóricos com aquilo que se vivencia e adquirindo olhares mais críticos quanto a situações que, infelizmente, repetidamente se tornam corriqueiras em espaços de cuidado. No âmbito da saúde mental, ainda que se tenham tido notórios avanços instituídos desde a promulgação da Lei Antimanicomial, tanto em âmbito federal quanto no Rio Grande do Sul, e de um olhar que coloca a saúde como um direito humano a ser assegurado, seguem-se percebendo práticas que não necessariamente buscam a promoção do cuidado em liberdade, territorial ou emancipatório, ao contrário, podem ser aliadas a práticas coercitivas e remanicomializadoras. Dentre elas, questiona-se o alto número de internações judiciais solicitadas aos usuários que possuem algum diagnóstico de transtorno psiquiátrico ou que façam uso abusivo de drogas, incluindo o público infanto-juvenil, como uma medida interventiva primária e/ou corriqueira (Rio Grande do Sul, 1992; Scisleski et al., 2008; Barison \& Gonçalves, 2016; Guareschi et al., 2016; Mattos \& Gregoviski, 2020; Leal et al., 2021; Gregoviski \& Ferreira, 2021; Santos \& Kuhn, 2021).

As ações de judicialização do cuidado, especialmente daqueles que fazem uso de substâncias psicoativas e/ou de crianças e adolescentes, estão em alta em um cenário no qual há uma disputa constante entre modelos biomédicos versus a proposta biopsicossocial. No Brasil, percebe-se um alto gasto orçamentário com processos de internação compulsória, ou seja, aquelas nas quais não há o consentimento do usuário, porém há uma leitura de necessidade pelo poder judiciário, ainda que contra a sua vontade. De tal forma, diante da conjuntura do país, da grande demanda por questões relacionadas aos problemas de saúde mental e das altas taxas de internações compulsórias infanto-juvenis, necessita-se abordar essa temática (Barison \& Gonçalves, 2016; Guareschi et al., 2016; Mattos \& Gregoviski, 2020; Leal et al., 2021).

Sobre o regime de internações psiquiátricas no Brasil, coloca-se que é algo previsto como uma forma de cuidado quando os recursos extra hospitalares são insuficientes, sendo elas: voluntária - consentida, involuntária - solicitação de terceiros -, e compulsória - determinada judicialmente (Brasil, 2001). Logo, não há, aqui, a intenção de se descartar internações compulsórias como uma possibilidade de cuidado, especialmente em situações de crises que colocam riscos a si ou terceiros, porém se critica o aumento exacerbado desses casos e o alto investimento financeiro em ações de judicialização do tratamento. 
Assim sendo, o objetivo deste artigo é o relatar a vivência de uma residente em saúde mental, que atuou em espaços de assistência à saúde e gestão, discutindo situações de judicialização do cuidado em crianças e adolescentes.

\section{Fundamentação Teórica}

A saúde mental se concretiza como uma política pública relativamente recente no país, conquistada pelo processo da Reforma Psiquiátrica brasileira (inspirado no movimento reformista italiano protagonizado por Basaglia). Assim, ainda na década de 30, o tratamento ao adoecimento psíquico ocorria por intermédio da institucionalização psiquiátrica permanente daqueles diagnosticados com alguma patologia mental, bem como de outros sujeitos considerados indesejados socialmente, como é o caso de pessoas negras, pobres, homossexuais, dentre outras. Com advento do movimento antimanicomial e com as diversas lutas estabelecidas por seus ativistas, desmantelam-se os espaços físicos dos manicômios, pautando o cuidado a partir de dispositivos que preconizam a (re)inserção social, tais como os Centro de Atenção Psicossociais (CAPS). Isto permitiu o redirecionamento do modelo de cuidado existente e criou uma Rede de Atenção Psicossocial (RAPS) que dá conta do acompanhamento integral e longitudinal daqueles que necessitam. Entende-se que, nesse cenário, há a formação de uma política pública em saúde mental no SUS que segue atuante até os dias atuais, ademais, percebe-se que isto possibilitou a ressignificação do sentido de loucura tido até então, que era o do usuário de saúde mental como um doente incurável, perigoso e criminoso (Borges \& Baptista, 2008; Carrara, 2010; Guimarães et al., 2013; Figueirêdo et al., 2014; Amarante \& Nunes, 2018).

No campo da infância e da juventude, porém, ainda se depara com processos nos quais a composição "justiça e saúde mental" opera na contramão tanto das diretrizes das políticas de saúde mental quanto nas do Estatuto da Criança e do Adolescente (ECA), que prezam o direito à dignidade humana, à liberdade e ao tratamento que não seja punitivo, cruel ou degradante. Nota-se um aumento crescente no número de processos de psiquiatrização seguidos de internação compulsória, principalmente em casos de adolescentes que estejam em conflito com a lei ou que sejam usuários de substâncias psicoativas, denotando um ferrenho olhar punitivista. Outrossim, percebe-se que a psiquiatrização desses jovens está condicionada a um alto consumo de psicotrópicos e múltiplos diagnósticos (Brasil, 1990; Vicentin, 2006; Leal \& Macedo, 2017; Costa \& Silva, 2017; Silva et al., 2019).

Agravando este cenário, os CAPS Infanto-Juvenis (CAPS IJ), referência no cuidado às crianças e aos adolescentes em sofrimento psíquico intenso, nem sempre conseguem dar conta dos atendimentos destes sujeitos por conta da desproporção existente entre a demanda e a oferta. Esta assimetria é presente em diversos países no mundo, porém é mais acentuada em nações subdesenvolvidas, como o Brasil, no qual a cobertura de CAPS IJ ainda se mostra baixa dentro da RAPS (Reis, 2012; Oliveira et al., 2018). Pensa-se que, entre outras possibilidades já sinalizadas aqui, isto pode ser um dos ocasionadores de uma maior tendência à judicialização do cuidado de crianças e adolescentes na atualidade.

Cabe a ressalva de que, infelizmente, muitas crianças e adolescentes em sofrimento ou adoecidos psiquicamente somente conseguem acompanhamento especializado quando chegam ao judiciário por outras vias mais agravadas, como atos infracionais, drogadição, violência e exploração sexual, trabalho infanto-juvenil, abandono de vulneráveis, situação de rua, mendicância e semelhantes. A saúde se torna, assim, outro direito dentre muitos que já foram violados. Essas violências e violações podem gerar sentimentos de desesperança e insegurança, além de estressores sociais que interferirão no desenvolvimento vital. Apesar de incipiente o que se sabe sobre as consequências das violações de direitos, pontua-se que podem estar relacionadas ao baixo rendimento, absenteísmo e abandono escolar, pensamentos de morte ou ideação suicida, comportamentos externalizantes e violentos, e abuso de drogas (Assis et al., 2009; Reis, 2012).

No caso de processos judiciais em prol da internação compulsória, percebe-se que se relacionam a ações da Defensoria ou Ministério Público por meio de denúncias de violações de direitos. Essa mobilização à judicialização ocorre, também, 
quando a própria família solicita de forma voluntária o apoio judicial para tal, inclusive diante de sintomatologias que não apresentam risco a si ou terceiros, como o caso de dificuldades escolares, comportamentais ou de automutilação. Assim, percebe-se que dentre as internações infanto-juvenis executadas, uma porcentagem altíssima se refere àquelas que advém de determinações judiciais (Reis, 2012).

Frisa-se a necessidade de uma linha de cuidado integral. Somente após todos os recursos esgotados é que devem ser utilizados laudos que evidenciam a gravidade do estado de saúde mental para justificar o tratamento via internação. Nesse sentido, os CAPS IJ têm a potencialidade de trabalho ao enfocar suas ações na singularidade dos sujeitos e seus contextos, com tratamentos que buscam articular rede intersetorial, território e familiares. Assim, há a possibilidade de melhora na qualidade de vida a longo prazo, desenvolvendo-se a cidadania, a autonomia, o fortalecimento de vínculos e a co-responsabilização entre os múltiplos atores que fazem parte do cuidado integral desses indivíduos em formação (Poletti, 2008; Franco \& Franco, 2010; Jorge et al., 2011; Reis, 2012).

Há, também, a necessidade de promoção de políticas intersetoriais para que se dê conta das questões de adoecimento psíquico no público infanto-juvenil, compreendendo-se que a saúde mental contempla múltiplos aspectos biopsicossociais. Para enfrentar essas questões, precisa existir articulação entre campos como a assistência social, justiça, educação e Conselhos Tutelares. Entretanto, pontua-se a existência de contradições nas ações que visam cuidado, mas despontam em coerções e punições. O controle disfarçado de cuidado se conecta diretamente com a ideia de biopoder proposta por Foucault, que coloca as características biológicas fundamentais dos sujeitos em uma política e torna o corpo público para ser controlado por quem tem mais poder. A partir das legislações, crianças e adolescentes se tornam objetos públicos a serem controlados pelo Estado enquanto não atingem a maioridade. Assim, há de se ter cuidado para que o diagnóstico não seja a porta de entrada para todo um sistema operacional de gerenciamento de suas existências, caucionando acesso ao serviço e submetendo ao processo de tratamento hospitalocêntrico como medida única (Foucault, 2008; Resende, 2008; Assis et al., 2009; Foucault, 2010; Franco \& Franco, 2010; Guareschi et al., 2015).

\section{Métodos}

Esta pesquisa se configura como um relato de experiência ao observar que o processo de aprendizagem ocorre a partir das vivências acadêmicas e profissionais, tendo-se um olhar crítico sobre elas para o aperfeiçoamento dos fluxos de trabalho. Compreende-se, assim, que relatos de experiência são ensaios acadêmicos-científicos capazes de provocar mudanças em cotidianos profissionais e em pesquisa científica (Mussi et al., 2021). Ademais, coloca-se que esta pesquisa possui delineamento qualitativo, transversal e exploratório, haja visto não serem inúmeros os estudos que abordam a proposta da RMS pensando o processo de judicialização do cuidado no público infanto-juvenil.

Como instrumentos, utilizaram-se diários de campo e observação participante. Os diários foram feitos com frequência diária afins de garantia de que a percepção da profissional residente pudesse se manter fidedigna do campo de atuação. As experiências documentadas ocorreram ao longo de dois anos do processo de especialização, pois a residente manteve contato com essa temática nos mais diversos campos pelos quais passou, destacando-se serviço de gestão em saúde e serviço de atenção psicossocial para usuários de substâncias psicoativas (inclusive aqueles que não tinham 18 anos completos).

Assim, além de discorrer sobre a experiência, discutiram-se os conceitos com aporte teórico clássico e contemporâneo, trazendo resultados de pesquisas com essa temática. No tocante à experiência e questões éticas pertinentes, destaca-se que seguiu os preceitos da Declaração de Helsinque e que foi um relato feito posteriormente à inserção, no qual não se trarão nomes de sujeitos ou instituições, visto que o foco é a vivência da autora em si. Logo, houve manutenção do sigilo e anonimato. $\mathrm{O}$ relato foi descrito e analisado a partir de seu conteúdo, conforme disposição a seguir. 


\section{Resultados e Discussão}

Um dos primeiros pontos assinalados nos diários de campo remete a caminhos possíveis diante da atuação compartilhada de casos com a RAPS. Há a percepção de que quanto mais forem possíveis tais articulações, menor serão os casos de crianças e adolescentes desacompanhados pelos serviços de cuidado que serão expostos a uma internação compulsória. Dá-se especial destaque para a potencialidade da aproximação entre o CAPS IJ com o CAPS Ad (a usuários de álcool e outras drogas), para apropriação e ampliação de ambos os espaços quanto a temática de uso de drogas em tenra idade. O diário de campo da residente coloca que a construção de um fluxo de cuidado esteve diretamente relacionada a uma preocupação dos gestores e dos trabalhadores da saúde com o extensivo número de sujeitos acometidos por tal situação, existindo um olhar crítico sobre seus papéis diante do cenário de crescente uso em idades cada vez mais precoces que, não raramente, podem levar a um abuso de drogas com consequências mais severas, especialmente quando essas crianças e adolescentes já eram alvos de violações de direitos pregressas. Além disso, vale destaque ao fato de que a RMS demonstra sua importância nesses espaços ao propor o início e incentivar a manutenção da educação permanente em saúde com os trabalhadores.

Porém, a experiência evidenciou que, ainda que existindo preocupação e articulações iniciais, os espaços dos CAPS por vezes se encontraram esvaziados. Há uma necessidade de se pensar em como os espaços de cuidado tradicionais podem atuar de forma criativa, inovadora e chamativa ao público infanto-juvenil, já que muitas vezes demonstram menor aderência ao cuidado proposto, inclusive pelas particularidades próprias de suas idades.

Mas a preocupação foi para além de como manter os usuários vinculados. Não é incomum se encontrar no diário de campo inquietações sobre a ausência de muitos profissionais do quadro técnico nas reuniões propostas para discussão de estratégias, pensando-se, inclusive, que uma possibilidade para tal seja a descrença quanto ao sucesso de estratégias de cuidado em rede, além de queixas habituais sobre a exaustão sentida pelo cenário precário e descaso de outros atores que deveriam estar afrente do cuidado, como outros pontos da rede ou familiares.

A desarticulação em rede não é fator recente de preocupação para o fluxo das políticas públicas, porém ainda se requer visibilidade com intuito de proporcionar maior crítica à lógica de encaminhamentos na qual muitas vezes os serviços se colocam. Por conta disto, há uma percepção, tanto teórica quanto prática, de que infantes e adolescentes "pipocam" de um serviço ao outro, sem que nenhum deles seja resolutivo de fato, portanto, encontrando-se em um limbo. Os encaminhamentos deveriam atuar na lógica de composição intersetorial, com ações multidisciplinar, territoriais, conjuntas e amplamente discutidas, sem embargo, não é o que ocorre (Battistelli \& Cruz, 2016).

Percebeu-se, ainda, que muitos dos adolescentes em situação de drogadição preferiram o acompanhamento pelo CAPS Ad, inclusive aqueles com uso esporádico e não prejudicial, proferindo maior identificação com o público, mesmo que, muitas vezes, as chacotas dos usuários adultos desencadeassem confrontos e crises. Uma queixa que emergiu foi a de infantilização que existia no serviço de atenção psicossocial que atendia crianças e adolescentes, denotando um acolhimento insuficiente diante de suas demandas. O pouco apoio familiar, muitas vezes presentes nos jovens acompanhados, refletia em abrigamento, situação de rua e vinculação com demais usuários de substâncias psicoativas, invariavelmente expondo-os a um agravamento de sua situação psicossocial e da drogadição. Constata-se a vinculação frágil existente entre esses jovens e demais atores (familiares, comunidade, escola), situação presente tanto nas observações práticas quanto em estudos que averiguaram o modo como se dá o cuidado a tais sujeitos (Scisleski et al., 2008).

Nesse sentido, assinala-se que a percepção tida pela residente, corroborada por muitos dos colegas, foi a de que havia um número excessivo de internações psiquiátricas compulsórias a adolescentes cujas principais demandas eram sociais, como os casos proferidos acima, de uso esporádico de substâncias. As articulações entre os serviços, ainda que iniciais, buscarem refletir acerca desse número, porém pouco se conseguia desenvolver, até porque, muitas vezes, as avaliações para internações 
sequer eram realizadas pelos profissionais dos CAPS, especialmente no caso do uso de substâncias. Nestas situações, não era incomum que se recebessem demandas judiciais de busca ativa e, ao se averiguar o estado de saúde do usuário, ele se encontrava internado em uma clínica ou comunidade terapêutica há meses. Tal ocorrência denota o quanto é impreterível que haja adequada fiscalização dos órgãos públicos e de conselhos profissionais para o cumprimento do fluxo de internação conforme o previsto por lei, em quaisquer que sejam as modalidade.

Além destes, a passagem da residente pela gestão proporcionou um maior esclarecimento diante dos fluxos consolidados na política de saúde mental em sua atuação ante processos judiciais de internação compulsória de crianças e adolescentes. Evidenciou-se a necessidade de que não fossem somente os trabalhadores dos CAPS que discutissem acerca dos aportes teóricos da infância e adolescência em paralelo à saúde mental, mas também os profissionais da gestão, por vezes alheios às propostas de cuidado e com acentuado e preocupante desconhecimento. Assinala-se mais uma vez a potência da residência enquanto dispositivo formador, tanto do acadêmico quanto dos profissionais dos espaços pelos quais passa ao longo dos dois anos de formação.

A formação perpassando espaço de assistência à saúde e de gestão em saúde evidenciou o uso questionável do dispositivo de internação psiquiátrica. Aquilo que era para ser utilizado como recurso último, após exaustivas tentativas de aproximação e cuidado intersetorial, era utilizado como ação primária ou frequente ao mesmo infante ou adolescente, especialmente em casos de desorganização social (comportamentos externalizantes, situação de rua, conflitivas com outros pares, risco de exposição à violência etc.) e de uso de drogas (mesmo quando não de forma intensa ou frequente). Ainda que todos esses aspectos tenham sido documentados e enviados ao Poder Jurídico, notou-se que todos os âmbitos demonstram baixa crítica quanto a esta situação, visto que pouco se foi feito para mudanças longitudinais (tanto na rede em si, quanto em casos individuais). Houve uma preocupação constante da residente na reflexão sobre como o Estado se vale de seu poder e trancafia pessoas pelo denominação de loucura.

A justificativa de internações prolongadas, acima do período estipulado pela legislação, era a de falta de acompanhamento da rede protetiva, situação que, algumas vezes, ainda que verídica, causa inquietação sobre como se fará possível mudar essa perspectiva. Vê-se que a psiquiatrização no campo da infância e da adolescência faz prevalecer os argumentos da proteção como legitimação de formas de coerção e de restrição arbitrária de liberdade (Vicentin et al., 2015). Com tais questionamentos, inquieta pensar que a mesma justiça que autoriza a internação dessas crianças e adolescentes é a mesma que durante suas vidas atuou de forma ineficaz e negligente.

Por fim, coloca-se que todas as vivências sinalizam para o fato de que a produção de atos de saúde somente será efetiva e garantida quando feita em rede, por todos os trabalhadores e atores de cuidado (Merhy, Feuerwerker \& Cerqueira, 2010). Percebeu-se que quanto maior era o engajamento dos serviços com as crianças e os adolescentes, maiores também eram os sucessos terapêuticos e a produção de saúde em ato.

\section{Considerações Finais}

Tradicionalmente, vê-se a perpetuação da ideia de que a família, sociedade e Estado asseguram os direitos humanos garantidos a crianças e adolescentes como prioridade. Porém, na prática é possível perceber um cenário bastante oposto. No caso da judicialização do cuidado com esse público, assunto preocupante e crescente na dimensão pública, questiona-se qual o limite que separa uma proposta de cuidado de uma violação de direitos. Faz-se necessário refletir sobre o porquê de políticas públicas não estarem sendo capazes de garantir direitos, visto a constante e crescente violação percebida, especialmente em tempos de aumento das vulnerabilidades sociais em decorrência do cenário macrossocial.

A defesa de uma saúde pública, universal e gratuita não pode ser deslocada da defesa de formas de cuidado emancipatórias, libertas, livre de discriminações e articuladas com os atores que fazem parte do cuidado quando se entende que 
a saúde vai muito além da ausência de doenças, dizendo respeito a todo o contexto em que se está inserido e como é a relação estabelecida com ele. A saúde mental enquanto política reformista não pode ficar omissa diante de práticas asilares e que não vão de encontro ao proposto na legislação pertinente, principalmente ao se discursar sobre sujeitos em pleno desenvolvimento. Precisa-se atentar para que as intervenções ditas de cuidado não provoquem mais danos psíquicos do que benefícios.

Acredita-se que para garantir cuidado infanto-juvenil efetivo não basta apenas articulações entre serviços especializados em saúde mental, mas a de todos os atores envolvidos, pensando território e políticas vigentes. Ressalta-se a urgência do aperfeiçoamento técnico daqueles que lidam com mandados judiciais e pouco conhecem sobre a forma de cuidado em saúde mental preconizada pelos movimentos reformistas. O papel do residente entra nesse sentido, potencializando discussões teóricas que se aliem às técnicas, promovendo reflexões críticas com análises participativas, percebendo incoerências e contradições na execução das políticas.

Finalmente, sinaliza-se que essa experiência contribuiu significativamente para o desenvolvimento profissional da residente, reforçando o quanto este tipo de formação é importante àqueles que visam a atuação no âmbito da saúde pública. Entre as inúmeras potencialidades possíveis, destaca-se a articulação com processos educativos da equipe, fomente de rede intersetorial e reflexo crítico ancorada na literatura. Assim, coloca-se a RMS como importante estratégia de construção de cuidado em saúde e políticas públicas coerentes com o cenário social. Sugere-se como agenda de pesquisa, estudos que versem sobre o olhar do público infanto-juvenil sobre o processo de judicialização do cuidado, bem como investigações que sigam aprofundando o potencial da RMS nesse cenário.

\section{Referências}

Amarante, P., \& Nunes, M. O. (2018). A reforma psiquiátrica no SUS e a luta por uma sociedade sem manicômios. Ciência \& Saúde Coletiva [online]. 23(6). Assis S. G. et al. (2009). Situação de crianças e adolescentes brasileiros em relação à saúde mental e à violência. Ciência \& Saúde Coletiva, 14.

Barison, M. S. \& Gonçalves, R. S. (2016) Judicialização da questão social e a banalização da interdição de pessoas com transtornos mentais. Serviço Social \& Sociedade [online]. 125

Battistelli B. M., \& Cruz L. R. (2016). Saúde Mental na Infância: cuidado e cotidiano nas políticas públicas. Revista Polis e Psique, 6(3).

Borges C. F., \& Baptista T. W. F. (2008). O modelo assistencial em saúde mental no Brasil: a trajetória da construção política de 1990 a 2004 . Cadernos de Saúde Pública, 24.

Brasil. (1990). Lei $n^{\circ} 8.068$, de 13 de julho de 1990. Dispõe sobre o Estatuto da Criança e do Adolescente e dá outras providências.

Brasil. (2001). Lei $n^{\circ}$ 10.216, de 6 de abril de 2001. Dispõe sobre a proteção e os direitos das pessoas portadoras de transtornos mentais e redireciona o modelo assistencial em saúde mental.

Brasil. (2005). Lei $n^{\circ} 11.129 / 05$. Institui as residências multiprofissionais e em área profissional da saúde.

Carrara, S. L. (2010). A História Esquecida: os Manicômios Judiciários no Brasil. Journal of Human Growth and Development, $20(1)$, 16-29.

Figueirêdo, M. L. de R., Delevati, D. M., \& Tavares, M. G. (2014). Entre loucos e manicômios: história da loucura e a reforma psiquiátrica no brasil. Caderno De Graduação - Ciências Humanas E Sociais - UNIT - ALAGOAS, 2(2), 121-136.

Foucault M. (2008). Segurança, Território, População. Trad. Eduardo Brandão. Martins Fontes.

Foucault M. (2010). Crise da medicina ou crise da antimedicina. verve. Revista semestral autogestionária do Nu-Sol., 18, 167-194.

Franco C. M., \& Franco T. B. (2010). Linhas do cuidado integral: uma proposta de organização da rede de saúde.

Gregoviski, V. R., \& Ferreira, L. A. dos S. (2021). Contando Histórias, Trilhando Caminhos: A experiência da residência em saúde mental no cuidado aos trabalhadores da saúde. Research, Society and Development, 10(14).

Guareschi, N. M. F, Ecker D. D., Souza F. M., \& Galarca L. G. (2015). Justiça e Saúde Mental: Internação, Tratamento e Alta de Adolescentes Usuários de Drogas. In: Scisleski A, Guareschi NMF (Orgs.), Juventude, Marginalidade Social e Direitos Humanos: da Psicologia as Políticas Públicas. (1).

Guareschi N. M. F., Lara L, \& Ecker D. D. I. (2016). A internação compulsória como estratégia de governamentalização de adolescentes usuários de drogas. Estudos de psicologia (Natal). 21(1), 25-35.

Guimarães, A. N. et al. (2013). Tratamento em saúde mental no modelo manicomial (1960 a 2000): histórias narradas por profissionais de enfermagem. Texto \& Contexto - Enfermagem [online]. 22(2), 361-369. 
Research, Society and Development, v. 10, n. 16, e175101623802, 2021

(CC BY 4.0) | ISSN 2525-3409 | DOI: http://dx.doi.org/10.33448/rsd-v10i16.23802

Jorge M. S. B. et al. (2011). Promoção da Saúde Mental-Tecnologias do Cuidado: vínculo, acolhimento, co-responsabilização e autonomia. Ciência \& Saúde Coletiva, 16, 3051-3060.

Leal D. M., \& Macedo J. P. (2017). A penalização da miséria no Brasil: os adolescentes “em conflito com a lei”. Textos e Contextos. 22.

Leal, F. X. et al. (2021). Gastos com internações compulsórias por consumo de drogas no estado do Espírito Santo. Saúde em Debate [online]. 45(129), 378392.

Mattos, A. C. E., \& Gregoviski, V. R. (2020). A Saúde Mental em Evidência: Narrativas de um Caminho Utópico. São Leopoldo: Casa Leiria.

Merhy E. E., Feuerwerker L. C. M., \& Cerqueira M. P. (2010). Da repetição à diferença: construindo sentidos com o outro no mundo do cuidado. Semiótica, afecção \& cuidado em saúde. 60-75.

Mussi, R. F. de F., Flores, F. F., \& Almeida, C. B. de. (2021). Pressupostos para a elaboração de relato de experiência como conhecimento científico. Práxis Educacional, 17(48), 60-77.

Oliveira S. M., Silva M. S., \& Nogueira M. C. A. (2018). Saúde mental infanto-juvenil: a necessidade de políticas públicas mais efetivas pelo Estado. Rev. Mult. Psic., 12(41).

Poletti P. C. (2008). Intersetorialidade e a Clínica em Saúde Mental: construindo e ampliando redes para a inclusão. Monografia.

Reis C. (2012). (Falência Familiar) + (Uso de Drogas) = Risco e Periculosidade: a naturalização jurídica e psicológica de jovens com medida de internação compulsória. Dissertação.

Resende C. C. F. (2008). Aspectos legais da internação psiquiátrica de crianças e adolescentes portadores de transtornos mentais. Revista Igualdade Edição Temática Drogadição, 41(32).

Rio Grande do Sul. (1992). Lei Estadual número 9.716 de 7 de agosto de 1992. Dispõe sobre a Reforma Psiquiátrica no Rio Grande do Sul, e dá outras providências [Internet]. Diário Oficial [do] Rio Grande do Sul.

Santos, M. F. R. dos, \& Kuhn, M. F. (2021). Saúde como direito humano: vivências do cotidiano no SUS. Research, Society and Development, 10(12).

Scisleski A. C. C., Maraschin C, \& Silva R. N. (2008). Manicômio em circuito: os percursos dos jovens e a internação psiquiátrica. Cadernos de Saúde Pública, 24.

Silva P. R. F. (2017). A atenção em saúde mental aos adolescentes em conflito com a lei no Brasil. Ciência \& Saúde Coletiva, 22.

Silva P. R. F, Gama F. L., \& Costa N. R. (2019). Atenção em saúde mental para adolescentes femininas em Unidades Socioeducativas: dilemas de governança e medicalização. Saúde Debate. 43(7).

Vicentin M. C. G., Assis D. A. D., \& Joia J. H. (2015). O direito de crianças e adolescentes ao cuidado em saúde mental: tensões entre proteção e tutela no caso do uso de drogas. DIKÉ Revista do Mestrado em Direito da UFS, 4(1).

Vicentin M. C. G. A questão da responsabilidade penal juvenil: notas para uma perspectiva ético-política. In.: ABMP, Secretaria Especial de Direitos Humanos, organizadores. (2006). Justiça, Adolescente e Ato infracional: socioeducação e responsabilização. Ilanud. 